\title{
Políticas urbanas y gobernabilidad de las ciudades: el «modelo francés»
}

\section{Christian Lefèvre*}

Francia es un Estado unitario, en el sentido de que, al men os hasta el momento, las leyes de la República se aplican en todo el territorio nacional aunque el actual gobierno acaba de autorizar la experimentación en julio de 2002, es decir, una interpretación local de la legislación nacional. La organización institucional francesa se fundamenta en el sistema napoleónico donde a cada nivel de entidad local (región, departamento, municipio) le corresponde una estructura desconcentrada del Estado, representada en el nivel de las regiones y los departamentos por la prefectura, en el nivel infradepartamental por las subprefecturas, y en el nivel de los municipios por los alcaldes, que son a la vez electos locales y representantes del Estado.

El proceso de descentralización se inició a mediados de los años 70 a través de una globalización progresiva de las subvenciones centrales hacia las entidades locales. Se concretó principalmente en las leyes de descentralización de 1982 y 1983, que transfirieron un gran número de competencias del Estado y de sus estructuras desconcentradas hacia los ejecutivos locales, principalmente los consejos generales en los departamentos y las alcaldías en los municipios. La única innovación institucional de las leyes de descentralización fue el establecimiento de las regiones como entidades regionales, lo que ya era una realidad en 1986 con la elección por sufragio universal directo de los ejecutivos regionales.

La cuestión de la gobernabilidad de las ciudades debe considerarse en este contexto político institucional porque los poderes públicos, y especialmente el Estado, son aún los dueños del juego, al menos en la elaboración y producción de las políticas urbanas, instrumento importante de la gobernabilidad de las ciudades. H ay, por lo tanto, un modelo francés que se caracteriza por un predominio del Estado, que interviene directamente con su aparato técnico-administrativo y con sus empresas y agencias, por una discreción de los actores económicos y sociales, y por el recurso a la reforma de las instituciones urbanas que están consideradas como las únicas capacitadas para resolver las cuestiones de gobernabilidad de las ciudades. Sin embargo, las transformaciones políticas, económicas y sociales actuales comienzan a cuestionar los fundamentos de este modelo.

\section{La gobernabilidad de las ciudades es una cuestión que compete en primer lugar a los poderes públicos}

En Francia, la gobernabilidad de las ciudades sigue siendo una cuestión que atañe exclusivamente a los poderes públicos, y especialmente al Estado. Se trata de un paradigma de las políticas públicas francesas, en las antípodas de la concepción norteamericana 0 británica. H ay, pues, dominio de los actores públicos y especialmente de las autoridades electas, que se traduce en una más débil - 0 al menos más discreta- presencia de otros actores económicos y sociales. 


\section{El dominio de las autoridades públicas}

El Estado francés mantiene un rol central innegable en el seno de la sociedad francesa, lo que se comprende por la historia de la formación del Estado-nación en el transcurso de los siglos, desde por lo menos el Antiguo Régimen. En efecto, el Estado se construyó contra los señores feudales, luego progresivamente contra las entidades locales y principalmente contra las ciudades. Receloso de la importancia de los upoderes» locales, estuvo durante siglos oponiéndose a toda veleidad de emancipación de las entidades territoriales que, por el contrario, ha tenido cuidadosamente encuadradas, controladas y dirigidas.

En consecuencia, las políticas públicas en el nivel local son históricamente en primer lugar las políticas del Estado. Para elaborarlas y conducirlas, ha construido un sistema técnico-administrativo importante, compuesto no solamente de organismos y estructuras en el interior mismo de los ministerios y de sus organismos desconcentrados, sino también de un gran número de empresas y de instituciones públicas específicas. Después de la guerra, el Estado creó la Caja de D epósitos y Consignaciones (CDC), institución pública administradora de los ahorros de los franceses, pero que sobre todo interviene, gracias a este recurso financiero colosal, en las políticas de desarrollo urbano (construcciones inmobiliarias, principalmente creación de equipamientos e infraestructuras). La CDC se ha asociado a las entidades locales para crear sociedades de economía mixta (SEM), cuyo capital es esencialmente, incluso totalmente, público, y que participan activamente en las políticas urbanas. En segundo lugar, el Estado interviene a través de organismos ad hoc, como las instituciones públicas de ordenación concebidas para desarrollar planes completos de las aglomeraciones (ciudad y sus extrarradios) francesas, y ello en cooperación más o menos intensa con las entidades territoriales. Por otra parte, el Estado sigue estando muy presente en las ciudades a través de sus sociedades nacionales como Electricidad y $G$ as de Francia, 0 como la Sociedad N acional de Ferrocarriles Franceses (SN CF), empresas importantes no sólo por sus actividades sino además por su patrimonio financiero.

D esde comienzos de los años 80, las entidades locales, sobre todo las más poderosas porque han sido las más beneficiadas por las leyes de descentralización, como las grandes ciudades y los departamentos, han intervenido a su vez de manera legítima en las políticas locales y principalmente en las políticas urbanas, sea de manera directa con sus inversiones, sea más indirectamente por medio de estructuras que controlan, como las agencias locales de desarrollo o los comités de expansión.

\section{La debilidad de la sociedad civil y de los otros actores sociales}

$M$ ientras que en los años 60 y 70, el Estado promueve la participación de las fuerzas vivas de los territorios en la creación institucional y en la formulación de las políticas públicas en el marco del desarrollo de las regiones, mientras que las le yes de descentralización de 1982 y 1983 consagran esta participación institucionalizándola en los consejos económicos y sociales regionales, los poderes públicos no habían juzgado adecuada su actuación en el nivel de las aglomeraciones. Esto no significa de ningún modo que los actores económicos y sociales estén ausentes de las políticas urbanas, sino que hasta hace poco su implicación era más bien escasa.

En Francia, el sector privado está muy presente por el simple hecho de que administra numerosos servicios públicos bajo el sistema de concesión (abastecimiento de agua, tratamiento de residuos, transportes públicos). I gualmente participa en numerosas sociedades de economía mixta en cooperación con las entidades locales. En cambio, lo mismo que los interlocutores sociales, son poco visibles en la elaboración de las políticas urbanas, en la producción de los documentos estratégicos y en las orientaciones de las inversiones. Las estructuras de representación de la sociedad civil, cámaras de comercio (CCI), uniones patronales, sindicatos, asociaciones, son invitadas (algunas por obligación legal como las CCI) a la elaboración de numerosos documentos (planes directores, planes de desplazamientos urbanos), pero su participación es modesta en los procedimientos que son ampliamente controlados por las autoridades públicas y especialmente las autoridades electas. Esto no significa, sin embargo, que las empresas, por ejemplo, no mantengan relaciones con los poderes públicos y que no hagan oír su voz, pero estas relaciones pasan por otros canales, más discretos, más informales, pero probablemente eficaces. Sin embargo, llega un momento en el que uno de los interlocutores, a veces los dos, desean dar a estas relaciones una forma más institucional izada, más visible. Las cámaras de comercio y de industria (CCI) han constituido durante mucho tiempo la estructura por la cual el sector privado participaba en los debates públicos y podían dar su opinión sobre proyectos urbanos y hacían pasar sus propios mensajes. La dé cada de los noventa vio nacer aś al gunas iniciativas sin marco legal en algunas aglomeraciones.

La sociedad civil siguió siendo un actor discreto de las políticas urbanas en una sociedad en la que lo público, y principalmente el Estado, mantiene la casi totalidad de la legitimidad de acción. Veremos más adelante que esta característica del «modelo francés» se dirige hoy en día hacia modificaciones significativas. 


\section{En Francia, es el Estado el que garantiza los equilibrios}

La ordenación del territorio ha sido uno de los sectores de las políticas públicas donde la hegemonía del Estado ha sido particularmente importante, hegemonía fundada sobre la autolegitimación de su rol en tanto que productor y garante de los equilibrios económicos y sociales en el nivel nacional. Este rol del Estado se inició desde principios de los años 60 con las grandes políticas de ordenación del territorio, como las relativas a las me trópolis de equilibrio, pero ha perdurado hasta hoy con la Ley de O rientación y de D esarrollo Sostenible del Territorio de 1995, y aún más recientemente con la Ley «Solidaridad y Renovación U rbana» (SRU) de 2000. En lo referente a las políticas urbanasy la gobernabilidad de las ciudades, la acción del Estado es significativa sobre todo en dos campos: el de la planificación y el de los equilibrios sociales, expresados por la «política de la ciudad». Es a estos dos temas a los que dirigimos nuestra atención ahora, aun cuando el Estado está interviniendo en otros, como el de la intermunicipalidad y el de la fiscalidad local, que trataremos en la tercera parte.

\section{Políticas urbanas coherentes con la planificación}

En 1967, período en el cual el Estado reina como dueño y señor en las políticas urbanas, se vota la Ley de 0 rientación Territorial (LOF), que va a servir de marco a la acción pública urbana hasta hoy. Esta ley fija para los decenios futuros los marcos jurídicos y los instrumentos de ocupación de los suelos. C rea aś los Planes Directores de Desarrollo y U rbanismo (SD AU), documentos al principio iniciados y aprobados por el Estado, pero hoy enteramente controlados por las entidades locales debido a la descentralización. Se trató al principio de instrumentos de localización de los equipamientos y de las infraestructuras que fijan en el término medio el desarrollo de las aglomeraciones, en la medida en que sus territorios de intervención buscan adaptarse a los territorios funcionales. En los SD AU debe darse forma a los Planes de 0 cupación de los Suelos (POS), documentos municipales encargados de establecer a nivel municipal las orientaciones contenidas en los SD AU. La LOF establece luego una jerarquía en los documentos de urbanismo dando la supremacía a los SD AU pero sin conferirles un status de oponibilidad jurídica.

La LOF establece igualmente una nueva estructura encargada de la planificación a nivel de las aglomeraciones: las agencias de urbanismo. Se trata de estructuras de investigación y de ase- soramiento dirigidas a las entidades locales, al principio aún controladas por el Estado, pero que van pasando progresivamente a manos de las entidades locales después de la descentralización. H oy existen agencias de urbanismo en la mayor parte de las aglomeraciones francesas. Su desarrollo ha jugado un importante papel en la capacidad técnica de las entidades locales en las políticas urbanas.

Pero el dominio del desarrollo urbano poco a poco es contrarrestado por una progresiva obsolescencia de los documentos de planificación iniciados por la LOF. M uy pronto, los Planes de 0 cupación del Suelo (POS) se han encontrado desfasados con unos Planes Directores basados en un crecimiento demográfico y económico que no se ha producido con la amplitud prevista. $H$ an aparecido otros problemas, como el declive industrial y sus efectos espaciales, tales como la multiplicación de los terrenos baldíos en el corazón de las aglomeraciones. El desfase entre los POS y los Planes D irectores se ha agravado debido a que los dos documentos principales de urbanismo estaban pensados para articularse de manera armónica. El gran periodo de revisión de los planes directores, iniciado a finales de los años 80 y que termina ahora, ha producido nuevos documentos de planificación sin continuidad real con los antiguos planes. Hoy, si numerosas aglomeraciones francesas disponen de planes directores, se trata en realidad de documentos de marketing territorial, donde el objetivo no es la localización de equipamientos e infraestructura, sino una imagen de las prioridades de desarrollo de la aglomeración sin contenido preciso y sin preocupación real de localización de las prioridades. En este marco, la articulación entre el POS y el SD AU no tiene mucho interés. Los dos documentos parecen entonces obsoletos, tanto en su concepción como en su cobertura espacial. En efecto, los nuevos planes directores para la mayoría no se adaptan a los territorios funcionales de las ciudades, pero siguen siendo tributarios la mayoría de las veces de los antiguos SD AU.

En paralelo a esta evolución, otros documentos de planificación han salido a la luz con sus especificidades y territorialidades propias: planes de desplazamientos urbanos, programas locales de vivienda, planes de protección de los espacios verdes, etc... han venido a ayudar a los documentos existentes, sin buscar de verdad una postura coherente. Brevemente, la planificación territorial considerada aporta la armonía en el desarrollo urbano cediendo poco a poco el lugar a una yuxtaposición de planes sin articulación entre ellos.

En este contexto, después de una decena de años de experimentos local es sin éxitos verdaderos, el Estado está interviniendo de nuevo para remediar las disfuncionalidades de la planificación urbana francesa. En diciembre de 2000, se promulgo la Ley «SOlidaridad y Renovación U rbana» (Ley SRU), que pone fin a un sistema de planificación instaurado por la LOF de 1967, y lo reemplaza por un nuevo sistema que aporta más coherencia. Así, la 
Ley SRU deroga los Planes D irectores y los Planes de 0 cupación del Suelo (POS), para remplazarlos por los Planes de Coherencia Territorial (SCOT) y los Planes Locales de U rbanismo (PLU).

Los SCOT sirven para definir la evolución de la aglomeración y las prioridades en materia de vivienda, comercio, zonas de actividad y transporte; mientras que los Planes Directores se dirigen esencialmente al destino del suelo sin tener en cuenta las otras políticas en el nivel de la aglomeración. LOS SCOT son, pues, documentos (y por lo tanto procedimientos de elaboración) que buscan articular las políticas que estaban previamente aisladas las unas de las otras debido a los planes espećíficos (planes de desplazamientos urbanos para los transportes, programas locales de vivienda para al ojamiento, etc.). No habrá pues a nivel de la aglomeración más que un documento único encargado de asegurar la coherencia territorial de las políticas públicas. Para hacerlo, el SCOT deberá apoyarse sobre «un proyecto de orde nación y desarrollo sostenible».

LoS PLU suceden a los POS. Presentan el proyecto urbano del municipio en materia de ordenación, de tratamiento del espacio público, de paisaje y de medio ambiente. M ás flexibles que los POS (permiten, en efecto, destinar muchos usos a una misma zona), deben como estos últimos ser compatibles con los SCOT.

\section{El Estado garante de los equilibrios sociales: la política de la ciudad}

El otro gran instrumento de intervención del Estado sobrela ciudad se denomina «la política de la ciudad». Ante la degradación de la situación social y económica de lo que es en primer lugar percibido como «el mal de los extrarradios», el Estado pone en marcha medidas de redinamización del tejido económico, social y físico urbano a partir de principios de los años 80 . H acia la mitad de los años 80, el Estado estableció la política del «D esarrollo Social de los Barrios». Sin parar pero con un paso vacilante, creó en 1988 la Dirección Interministerial de la Ciudad (DIV) que se colocó a partir de 1990 bajo la responsabilidad del primer ministro de la ciudad. En 1992, se creó en el nivel local una administración desconcentrada encargada muy especialmente de la política de la ciudad: el «subprefecto de la ciudad». Para el establecimiento de estas tres innovaciones institucionales, el Estado buscó crear luego un nuevo enfoque multisectorial, integrado, de su acción. El M inisterio de la Ciudad es, en efecto, no sectorial. Está simbolizado por su primer ministro, M. D elbarre, ministro de Estado con más voluntad que presupuesto efectivo, puesto que la idea existente es cubrir las medidas que se tomen con los recursos provenientes de los diferentes ministerios (Asuntos Sociales, Interior, 0 bras Públicas y Transporte). La creación de la DIV sigue la misma lógica. En los pri- meros años, la DIV no dispone de presupuesto propio, sino que se alimenta de los presupuestos de los diferentes ministerios.

La política de la ciudad será para el Estado la ocasión para una nueva innovación de tipo organizativo en el medio urbano. Consciente de su incapacidad de asumir solo las políticas necesarias para responder a los problemas de los extrarradios, debido principalmente a la descentralización y a la crisis de las finanzas públicas, el Estado aplica a la ciudad una nueva forma de hacer políticas públicas por medio de la contractualización, establecida en el marco de los primeros Contratos del Plan Estado-Regiones (CPER) entre 1984 y 1988. Los CPER establecían, en efecto, una asociación entre las entidades territoriales y el Estado para los temas de planificación y ordenación del territorio. La gestión es descentralizada y contractual; está dirigida por las regiones en favor de las entidades locales. En el marco de la política de la ciudad, las autoridades públicas (esencialmente el Estado a través del M inisterio de la Ciudad y los municipios implicados) se ponen de acuerdo sobre los obje tivos y las posibilidades de las medidas a emplear en el marco de un documento específico firmado por los interlocutores. $\mathrm{N}$ acen entonces, a principios de los años 90 , los primeros «contratos de ciudad», cuya gestión se repetirá luego en muchos otros campos y que se perpetúa hasta hoy.

Aunque innovadora, esta gestión se desarrolla con un sistema de actores limitado al sector público. Incluso cuando los círculos asociativos son consultados, no participan directamente en la elaboración de las políticas y de las acciones. Intervienen sólo más adelante, aportando recursos, informaciones y capacidad de diagnóstico. Lo mismo ocurre en el sector del desarrollo económico, que la política de la ciudad emplea marginalmente y donde las empresas sólo están implicadas débilmente. Si hay innovación, se limita a la esfera pública y, además, en el nivel local; por ejemplo, la creación de las M isiones de D esarrollo U rbano, especies de grupos de reflexión y de la aplicación de la política de la ciudad, compuestas por profesionales de orígenes diversos (sociólogos, arquitectos, economistas, urbanistas) que trabajan juntos en sectores hasta ahora completamente separados. Lo mismo ocurre con los contratos de la ciudad, donde los comités de dirección están dominados ampliamente por los actores públicos, fundamentalmente el Estado desconcentrado y las entidades locales, sobre todo los municipios.

Pero la intervención estatal en la política de la ciudad toma otra amplitud a partir de los años 90 y culminará en la Ley sobre la «Solidaridad y la Renovación U rbana» (Ley SRU) en el 2000. En efecto, en 1991, el Estado promulga la «Ley de O rientación de la Ciudad» (LOV), que sirvió en 1996 para el «Pacto de reactivación de la ciudad». D etrás de estas denominaciones se esconden toda una serie de medidas para tratar de contener la crisis social y económica que estalló en los extrarradios franceses en esa época. Esas medidas, como los contratos de ciudades, fueron 
muy discutidas y los analistas más críticos denunciaron una lista de medidas mal integradas que no supieron hacer una verdadera política. No es este artículo el lugar adecuado para hacer una crítica, lo importante es que el Estado francés, a semejanza de su homólogo británico, pero al contrario que la mayoría de los otros Estados europeos, considera que la «cuestión urbana» es de su incumbencia. La promulgación de la Ley SRU en diciembre de 2000 es el último ejemplo.

El primer objetivo de la Ley SRU, el más polémico y que le da su nombre, es favorecer la mezcla social en la vivienda sancionando financieramente la negativa de al gunos municipios a recibir viviendas sociales. La meta es un reparto mejor de las viviendas sociales entre los municipios, imponiéndoles que dediquen al menos un $20 \%$ de su parque inmobiliario a viviendas sociales. 0 tras medidas están dirigidas a favorecer los transportes públicos y a promover un modelo urbano de fuerte densidad, a fin de reducir la segregación socio-espacial.

\section{La fragmentación institucional, el problema mayor de la gobernabilidad de las ciudades}

Las aglomeraciones, como territorios funcionales, han estado en el punto de mira de las leyes de descentralización. En efecto, los grandes vencedores de la descentralización francesa han sido los municipios y los departamentos y la reforma político-institucional de principios de los años 80 ha «olvidado» estos territorios. D esde entonces, la fragmentación institucional del territorio, y de las aglomeraciones en particular, está siendo el problema central de la gobernabilidad de las ciudades y, por tanto, de las políticas urbanas. Esta afirmación no es un juicio de valor ni un diagnóstico pertinente de los problemas de gobernabilidad que atraviesan las ciudades francesas. Se podría contestar muy claramente que la fragmentación institucional no es el problema central. Pero no es menos cierto que lo principal de la clase política y la mayor parte de los actores económicos y sociales la consideran el problema número uno, y por eso está en la agenda política desde hace una decena de años. D esde entonces, todas las políticas urbanas y las reformas institucionales han intentado mitigar este «olvido» de las leyes de descentralización, esperando actuar así a favor de una mejor gobernabilidad de los espacios urbanos.

\section{La fragmentación institucional y las políticas urbanas}

Francia es conocida por su fragmentación municipal. Con más de 37.000 municipios para 60 millones de habitantes y una superficie de 551.000 km², cuenta ella sola con tantos municipios como los otros países de la Europa de los quince. D esde principios del siglo XX, se ha favorecido la cooperación entre municipios a fin de mitigar la fragmentación. Durante años, la panoplia de estructuras intermunicipales se ha ido haciendo más amplia y compleja, hasta el punto de que hoy esta cooperación es vista paradójicamente como un refuerzo de la fragmentación institucional.

La cooperación intermunicipal es ejercida esencialmente por medio de estructuras que se han reagrupado bajo el término de Establecimientos Públicos de Cooperación Intermunicipal (EPCI). Esto significa que no se trata de entidades locales, porque sus competencias son específicas, y que los EPCI no están dirigidos por personas directamente elegidas por la población. Son los municipios los que envían sus propios representantes a ocupar un puesto en los consejos de los EPCI. La M ancomunidad Intermunicipal de Fin Ú nico (SIVU 1), la M ancomunidad Intermunicipal de Fines M últiples (SIVO M ), consorcios, distritos, comunidades urbanas, comunidades de municipios, comunidades de ciudades, comunidades de aglomeraciones (ciudad y sus suburbios), Sindicato de Aglomeraciones N uevas (SAN ), son las EPCI que forman la variedad de la intermunicipalidad francesa.

La intermunicipalidad francesa es cuantitativamente importante. En 1972 se han contado no menos de $10.636 \mathrm{EPCl}$. En 1999, los municipios se agrupaban en más del doble, puesto que había no menos de 20.184 estructuras intermunicipales. La fórmula de los SIVU es la más importante con gran diferencia. En efecto - y se trata de la segunda característica de la intermunicipalidad francesa- los agrupamientos se realizan de manera voluntaria. Los municipios escogen libremente participar o no en una estructura intermunicipal en nombre de la sacrosanta libertad municipal. No es pues sorprendente que hayan preferido agruparse con fórmulas menos constrictivas, como son los SIVU o los SIVO M. Precisemos que no se trata de un efecto de la descentralización; la libertad municipal existía ya antes de las leyes de 1982 y 1983, aunque éstas han reforzado la autonomía municipal.

A finales del siglo xx, hay aún más de 20.000 estructuras intermunicipales, que han venido a añadirse a los 37.000 municipios. Contribuyen así a fragmentar el territorio, pero parece que los espacios funcionales que son las aglomeraciones no disponen delos EPCI que los estructuren de manera adecuada. 
La fragmentación de los territorios es también el resultado de la acción del Estado. En efecto, las políticas estatales, que se han multiplicado en el marco de una contractualización en los diversos niveles de entidades, han conducido a una proliferación de divisiones territoriales, terminando con una verdadera fragmentación de la acción pública. Zonas francas urbanas de la Ley de 0 rientación de la Ciudad, G randes Proyectos U rbanos, Zonas de Educación Prioritaria (ZEP) del Ministerio de Educación N acional, territorios de los contratos de la ciudad, programas locales de vivienda (PLH) por citar algunos, a los cuales han venido a añadirse las zonas de las políticas europeas, han aumentado la fragmentación de los territorios urbanos.

La autonomía municipal y el sistema de fiscalidad local contribuyen a su vez a aumentar los efectos de la fragmentación, fomentando la competencia entre los municipios. El periodo de crecimiento y de competitividad territorial actual, exacerbado por los procesos de descentralización, ha hecho de los recursos fiscales un reto importante para los municipios. El impuesto profesional sobre todo, principal ingreso fiscal municipal, ha sido objeto de una competencia importante entre los municipios de una misma aglomeración. En efecto, este impuesto cobrado a las empresas lleva a cada municipio a intentar atraer a su territorio al máximo de empresas, jugando con la tarifa del impuesto, porque mientras más bajo sea el impuesto, más inclinadas se sienten las empresas a establecerse en el municipio referido; y cuanto más numerosas sean las empresas, más puede permitirse el municipio bajar su tarifa del impuesto profesional, atrayendo los ingresos suficientes para que contribuyan a hacer su territorio aún más atractivo para las empresas; y así sucesivamente. En este contexto, los municipios se han lanzado a una competencia, algunas veces feroz, en los años 80 y principios de los 90, creando de manera desordenada zonas de actividades susceptibles de atraer a las empresas. Ello ha provocado un despilfarro del dine ro público, una multiplicación de las zonas de actividad industrial o de la artesanía en una misma aglomeración, haciéndose la competencia entre ellas para atraer a las mismas empresas. En los años 90, se han multiplicado los informes de los poderes públicos y las cámaras de comercio que denuncian este efecto perverso del sistema fiscal y de la descentralización, subrayando la falta de cooperación entre los municipios y el foso que se crea en el seno de la ciudad entre municipios ricos, que pueden atraer a las empresas más rentables y menos contaminantes, y municipios pobres, condenados a perder empleo y actividades.

\section{2. ¿La cooperación entre entidades locales, solución al problema de la gobernabilidad de las ciudades?}

El cuadro intermunicipal esbozado a principios de los años 90 va a encontrarse cada vez más en falso con los cambios eco- nómicos, sociales y medioambientales. En los años 60 y 70, el debate sobre la adecuación entre territorios funcionales y territorios institucionales se produce esencialmente sobre cuestiones de infraestructuras y equipamiento y sobre la gestión de los servicios. La cuestión de las economías de escala y de la eficacia de los servicios era central. D esde mediados de los años 80, otros elementos se convierten en esenciales, se trate del desarrollo económico, de la protección del medio ambiente, de la lucha contra la pobreza y la exclusión social o de la solidaridad territorial.

D os leyes recientes de 1999 se han dirigido a la reducción de la fragmentación institucional desde el punto de vista de los diversos campos de las políticas públicas. Se trata de la Ley sobre el Reforzamiento y la Simplificación de la Cooperación Intermunicipal y la Ley sobre la O rdenación del Territorio.

La Ley sobre el Reforzamiento y la Simplificación de la C ooperación Intermunicipal fue seguida de numerosas leyes sobre la cooperación intermunicipal, promulgadas después de las leyes de descentralización. La ley puso en marcha una reestructuración institucional de la cooperación intermunicipal pretendiendo, como su título indica, simplificar la compleja organización de la intermunicipalidad francesa, donde al menos 9 tipos de EPCI coexisten, cada una con competencias, territorios y recursos diferentes. Esta ley, Ilamada «Ley C hevènement» nombre del ministro de Interior de la época, creó dos nuevos EPCI que debían con el tiempo cubrir espacios de solidaridad a partir de la puesta en marcha de un proyecto común de desarrollo económico y de ordenación. Progresivamente estos dos EPCI remplazarán por transformación automática a los existentes. Para permitir una mejor armonía económica y social en las aglomeraciones, la ley instauró también de manera obligatoria un impuesto profesional único (taxe professionnelle unique - TPU - ) sobre el territorio de las comunidades urbanas y de las comunidades de aglomeración, que representaban de hecho a los territorios urbanos, mientras que las denominadas comunidades de municipios se dirigían principalmente a las zonas rurales. Gracias a esta ley, las aglomeraciones francesas deben disponer progresiva pero rápidamente de una organización político-institucional simplificada, más clara para los habitantes, y de recursos financieros compartidos en el nivel del territorio funcional de la ciudad.

La lógica de creación de estos dos nuevos EPCI está en parte ligada a la reforma de las definiciones estadísticas del territorio francés por el Instituto N acional deEstadística (IN SEE). En efecto, hacia mediados de los años 90, el IN SEE propuso nue vas nociones estadísticas que permitiesen recoger mejor la evolución espacial y económica de las ciudades y los pueblos. Las nuevas nociones - aglomeración, polo urbano, área urbanase vinculaban más a las relaciones económicas y sociales entre los municipios que a la densidad o a la continuidad del espacio construido, como ocurría con las nociones anteriores. La Ley Chevènement se inscribe en este contexto, Así, las comunidades 
urbanas son «EPCI que agrupan muchos municipios formando un conjunto de más de 500.000 habitantes y que se asocian en el seno de un espacio de solidaridad, para elaborar y conducir juntos un proyecto común de desarrollo urbano y de ordenación de su territorio» (artículo 5). Las comunidades de aglome ración son «EPCI que agrupan muchos municipios y forman un conjunto de más de 50.000 habitantes al rededor de uno 0 más municipios centrales de más de 15.000 habitantes» (artículo 1). Su perímetro «puede extenderse a los municipios cuya inclusión en el perímetro comunitario es necesario para asegurar la coherencia espacial y económica, así como la solidaridad financiera y social necesarias para el desarrollo de la comunidad de aglomeración» (artículo 2). Así, la Ley Chevènement creó los EPCI cuyo perímetro corresponde en la lógica de su creación a las aglomeraciones (comunidades de aglomeraciones) y a las áreas urbanas (comunidades urbanas), definidas como espacios de solidaridad económica y social.

La justificación de la creación de las comunidades urbanas y de las comunidades de aglomeración se pone de manifiesto en las competencias que se les han concedido y los recursos fiscales y financieros de los que disponen. Así, la comunidad de aglomeración tiene como competencias obligatorias el desarrollo económico, la ordenación del territorio, el equilibrio social de la vivienda y la política de la ciudad, que son de interés comunitario. Se añaden cinco competencias opcionales entre las cuales deben escoger tres para asumirlas: saneamiento, agua, vías públicas, medio ambiente y calidad de vida, y equipamientos culturales y deportivos. La comunidad urbana posee seis competencias obligatorias: las de las comunidades de aglomeración, a las que hay que añadir la gestión de los servicios de interés colectivo (saneamiento y agua, cementerios, mataderos, servicios contra incendios), el desarrollo social y cultural y la protección y mejora del medio ambiente aś como la política de calidad de vida. En el plano fiscal y financiero, la Ley Chevènement estableció las me didas siguientes. LaTPU es obligatoria para las comunidades de aglomeración y las comunidades urbanas. El Estado se compromete también a entregarles una D otación Global de Funcionamiento (DGF, la principal subvención estatal a las colectividades locales) más grande.

La segunda ley, la Ley de 0 rientación para la 0 rdenación y el D esarrollo Sostenible del Territorio (LOADDT), Ilamada Ley Voynet, nombre del ministro de M edio Ambiente de la época, tiene un importante marchamo de «aglomeración» que se declina en tres términos: proyecto de aglomeración, consejo de desarrollo y contrato de aglomeración, que el Estado resume con una fórmula lapidaria: «un territorio, una estrategia, un proyecto, un contrato».

Dos elementos están en el centro de la LOADDT: el proyecto de desarrollo y la contractualización. La LOADDT señala lo que el Estado entiende por proyecto de desarrollo. «D etermi- na, por una parte, las orientaciones que establece la aglomeración en materia de desarrollo económico y de cohesión social, de ordenación y de urbanismo, de transporte y de vivienda, de política de la ciudad, de política de medio ambiente y de gestión de los recursos según las recomendaciones inscritas en las agendas 21 locales del programa "Acciones 21"... y, por otra parte, las medidas que permiten la puesta en marcha de esas orientaciones» (artículo 26). Yendo más lejos y completando la Ley Chevènement en ese sentido, la LOADDT especifica que si las aglomeraciones desean ser ayudadas financieramente por el Estado, «deberán constituirse en EPCI de TPU de al menos 50.000 habitantes». Podrán así beneficiarse de contratos particulares (contratos de ciudad, contratos de aglomeración) que serán incluidos en los contratos del plan Estado-Regiones, documento de programación que especifica las infraestructuras, equipamientos y medidas financieras de manera compartida entre el Estado y las regiones.

El territorio contemplado es el territorio considerado pertinente para las políticas públicas estratégicas. Se trata del territorio de las 140 áreas urbanas francesas identificadas en 1997 por el IN SEE y que definen los espacios funcionales urbanos. Esos territorios son igualmente los espacios de referencia de la Ley Chevènement, lo que hace que exista una línea directa entre la Ley sobre la Cooperación Intermunicipal y la Ley sobre la 0 rdenación del Territorio. La estrategia remite a los objetivos y a las prioridades decididas por la aglomeración, es decir, por la o las estructuras institucionales que la aplican. La puesta en escena de esta estrategia se efectúa en el marco de un proyecto, y es principalmente sobre este plano cómo la ley innova, vinculando la ayuda del Estado a la existencia de un proyecto de aglomeración.

La LOADDT necesita, en efecto, que «el proyecto de aglomeración sea un proyecto de desarrollo fundado sobre una reflexión interdisciplinaria y que descanse sobre un diagnóstico compartido por la mayoría de las fuerzas vivas del territorio en cuestión». Este proyecto debe «definir las orientaciones a medio y largo plazo fundadas sobre una identificación precisa de las políticas públicas a ejecutar y de las iniciativas privadas a fomentar». Sólo cuando este proyecto está elaborado y aprobado es cuando el Estado puede ayudar financieramente a la aglomeración, firmando con ella un contrato plurianual, llamado contrato de aglomeración. La manera de asegurar una coherencia tanto del contenido como de los medios financieros para poner en marcha el proyecto es que el contrato de aglomeración no sea un documento aislado, sino que por el contrario esté integrado en el contrato del plan que el Estado firma cada seis años con la re gión (CPER). Por este concepto, la región firma igualmente el contrato de aglomeración. Estableciendo un proyecto de aglomeración y firmando un contrato de aglomeración con el Estado, el área urbana se compromete - si no la tiene ya - a dotarse en los años siguientes de una estructura política, bien comuni- 
dad urbana 0 bien comunidad de aglomeración, según el tamaño de la aglomeración en cuestión.

La LOADDT innova igualmente creando los consejos de de sarrollo. Se trata de instancias obligatorias pero consultivas, que reúnen a electos, medios socio-profesionales y asociativos del territorio del área urbana. Estos consejos deben ser obligatoriamente consultados para la elaboración, aprobación y ejecución del proyecto de aglomeración. La ley no impone ninguna organización a los consejos de desarrollo. D eja, por el contrario, libertad de organización a los actores locales.

Es difícil hacerse una idea hoy en día de los consejos de desarrollo, porque la ley es muy reciente y la mayor parte de las aglomeraciones están en vía de constituirlos; pero la libertad concedida a las entidades locales evidencia que se va a producir una gran diversidad. Como ejemplo, el consejo de desarrollo de la comunidad urbana de Burdeos, uno de las primeros en ser creados, es re lativamente modesto (41 miembros en total, representando a los medios económicos, sociales, al movimiento asociativo, a las entidades locales y a los habitantes, que tienen 12 escaños) mientras que el del Gran Lyon es más grande (no menos de 500 miembros). La diversidad se manifiesta también en la importancia concedida a estos consejos. Mientras que el de Burdeos parece moderadamente activo, no ocurre lo mismo en Lyon.

Las leyes de 1999 parecen conseguir un verdadero éxito, principalmentela Ley «C hevènement». En efecto, las comunidades de aglomeración se han multiplicado y hoy la mayor parte de las grandes ciudades 0 de las ciudades medianas poseen un
$\mathrm{EPCI}$ de impuesto profesional único. En cambio, en relación con los contratos de aglomeración, el balance es por ahora modesto, con aproximadamente tres contratos firmados entre las entidades locales y el Estado a principios de 2002. En cuanto a sus contenidos, se continúan privilegiando las infraestructuras y los equipamientos, principalmente el transporte.

\section{Conclusión}

El «modelo francés» de políticas urbanas y de gobernabilidad de las ciudades, basado en el predominio de los poderes públiCOs, en un discreto papel de los actores económicos y sociales y que coloca a la construcción institucional como instrumento central de la gobernabilidad parece, pues, confortado por las tres ultimas grandes leyes de 1999 y de 2000 (Ley SRU). Estas tres leyes abren sensiblemente el sistema de actores y conceden, en un sentido amplio, un lugar más importante a la sociedad civil. Esto se confirmó por el voto en febrero de 2002 de la Ley «de mocracia y proximidad», que obliga a los municipios y aglome raciones de más de 80.000 habitantes a dotarse de comités de barrios, debiendo ser consultados en un gran número de ámbitos, principalmente la ordenación y el urbanismo. El «modelo francéss, tal y como se describe en este artículo, está en proceso de transformación, pero su evolución no parece, por ahora al menos, que ponga en entredicho sus propios fundamentos.

Notas

* Profesor de la Universidad de París VIII Val-La-M arnée, Profesor del Instituto Francés de U rbanismo y miembro del Laboratorio Techniques, Territoires et Sociétés (LATTS).

La traducción española de la versión original francesa ha sido realizada por $C$ armen
Pineda N eBot, Investigadora del Departamento de Ciencia Política y Relaciones Internacionales de la U niversidad Autónoma de M adrid.

1 N ota del traductor: SIVU son las siglas francesas de Syndi cat Intercommunal à Vocation U nique, y SIM O V las de Syndicat Intercommunal à Vocation M ultiple. 